\title{
PERCEPCIÓN DE LA INSERCIÓN A LA EDUCACIÓN POR PARTE DE ADULTOS MAYORES DE LA LOCALIDAD DE SUBA
}

\author{
Ruby Maritza Gerena $^{86}$
}

RESUMEN

El presente estudio tuvo como objetivo conocer la precepción sobre aprendizaje que tiene un grupo de adultos mayores de la localidad de Suba en este momento de la vida, para comprender qué les gustaría estudiar. Se realizó con 30 de ellos y se aplicó un enfoque cualitativo de tipo descriptivo, con técnicas como la entrevista, el grupo focal y la observación directa, para recolectar la información. A partir de la base referencial en Freire y Morín, Montero, y los procesos de aprendizaje como temáticas fundamentales para interpretar los resultados obtenidos, se llegó a la conclusión de que reconocer el interés del adulto mayor en un proceso de aprendizaje en la educación informal y para el trabajo, que sea lucrativa para beneficio propio y de sus familias.

Palabras clave: percepción, educación, adulto mayor, cognición.

\begin{abstract}
The present study had as aim knows the perception that has a group of major adults of the locality of Suba at this moment of the life, to understand what they would like to study. It was realized by 30 of them and there was applied a qualitative approach of descriptive type, with technologies as the interview, the focal group and the direct observation, to gather the information. Base on Freire and Morín, Montero, and the learning processes like fundamental subject matters to interpret the obtained results, giving as conclusion the recognition of the interest of the major adult in a learning process in the informal education and for the work, which should be lucrative for own benefit and of his families.
\end{abstract}

Key words: perception, education, elderly, cognition

\section{INTRODUCCIÓN}

La presente investigación da cuenta del proceso investigativo llevado a cabo sobre la percepción de la educación en el adulto mayor, ya que es de gran transcendencia a nivel cultural y social, a través de un proceso permanente de aprendizaje sin límite de edad, cultura, raza y sociedad, que el ser humano sea tenido en cuenta y valorado socialmente, por ese potencial de desarrollo que aún posee.

El contexto de la investigación fue establecido en un macro-contexto: El semillero Sofistas de Plata, 2009 y un micro-contexto, la localidad 11 de Suba al norte de la ciudad de Bogotá,

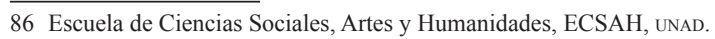


donde se pudo interactuar con el grupo de adultos mayores requeridos. El planteamiento del problema expresa la necesidad que hay en una población que no estudia, argumentación basada en las teorías de Freire citado por (Araujo y otros 1964), los cuales resaltan la importancia de la educación de personas adultas, para que los seres humanos sean libres, emancipados socialmente.

De tal forma que se plantea la necesidad de una educación incluyente a partir del planteamiento del adulto mayor frente a sus deseos y necesidad de logro, que se busca, surjan, desde la percepción del adulto mayor y no desde la visión de un investigador ajeno a esta realidad.

\section{JUSTIFICACIÓN}

El propósito de esta investigación es continuar indagando temas desde la Psicología Social Comunitaria relacionados con el adulto mayor, en este caso conocer su percepción de la educación en esta etapa de su vida, para la cual se inició con la exploración del discurso en un grupo de adultos mayores de la comunidad de Suba.

Realizar esta etapa investigativa permite tener miradas diferentes que aporten al conocimiento de temas como la educación del adulto mayor: es pertinente con el quehacer de la Psicología en especial el área social comunitaria según Montero (1984), es una práctica transformadora a través de la acción, cuyo objeto de estudio es la comunidad y el desarrollo comunitario, siempre está en continua búsqueda de identidad cultural, tiene vida propia y necesidades a nivel social y siempre se ha interesado por el bienestar de las colectividades y contribuye a disminuir el aislamiento social de la población más sensible a la vulneración psicosocial.

\section{Estudios relacionados}

Estudios en América Latina demuestran que es muy importante tener en cuenta la relación de la educación con las funciones cognitivas y las necesidades de aprendizajes en esta edad. La investigación de la "Relación entre Educación, envejecimiento y Deterioro Cognitivo" realizada en Arequipa, Abarca (2008), demuestra claramente que los adultos mayores con mejor nivel educativo tienen mejor nivel cognitivo, que aquellas personas que no están vinculadas en ningún tipo de educación. En la investigación "Percepción del aprendizaje desde el adulto mayor" realizada en Cuba (Agramonte y otros 2002), se demostró que en los adultos mayores no existen pérdidas ni deterioros significativos en las capacidades psíquicas, pero que sí existían necesidades de aprendizaje.

Asimismo es relevante tener en cuenta el concepto de andragogía (considerada arte y ciencia de ayudar aprender a los adultos). Alcalá (1997), inmerso en la educación permanente, se desarrolla en una práctica fundamentada en principios de participación (acción en tareas determinadas) y horizontalidad, (relación entre iguales apoyo mutuo, hasta donde sus potencialidades se lo permitan) para incrementar el pensamiento, la autogestión, calidad de vida y creatividad del participante adulto, cuyo propósito es proporcionarle oportunidades a los adultos para lograr su autorrealización personal y que comprende desde los 18 años hasta 
la edad mas tardía. Siendo estos los dos fundamentos de mayor importancia en la praxis andragógica.

Finalmente, realizar esta etapa investigativa con el grupo de adultos abre caminos para el conocimiento y abordaje de temas que conciernen al bienestar del adulto mayor y ayuda a mejorar su calidad de vida, tanto sociológica como psicológica ya que no sólo es un beneficio para el adulto mayor, sino para toda aquella población que está en preparación para esta etapa, que promuevan un trabajo por una cultura educativa en sus proyectos de vida, ya que esto generaría a largo plazo un mejor nivel cognitivo y salud mental para el adulto mayor de nuestra población colombiana.

\section{Contexto de la investigación}

El presente estudio surge de la línea de trabajo que lleva desde hace tres años el semillero de investigación Sofistas de la edad de plata en su trabajo con adulto mayor. En esta etapa investigativa se hizo un trabajo con la comunidad de Suba localidad 11 Bogotá, con un grupo de 30 personas estratos 2 y 3 con edades entre 59 y 79 años, con el fin de indagar la percepción que tienen sobre la educación en este momento de sus vidas. El grupo demostró su colaboración para responder la entrevista propuesta, donde cada uno relató a través del discurso lo que pensaba sobre educación, asimismo lo que les gustaría tener la oportunidad de aprender, con el fin de seguir siendo persona activas social y culturalmente.

\section{Planteamiento del problema}

Conocer la percepción que tienen sobre la educación en este momento de sus vidas es importante para entender qué es lo que a ellos les gustaría aprender, reflexionando sobre el hecho que la educación genera en ellos bienestar para su calidad de vida, su familia y la comunidad en general. El ser humano es visto desde una dimensión integral y se sabe que al reconocer sus fortalezas y debilidades permite enfocar la investigación desde lo social comunitario. Teniendo en cuenta a Freire citado por Araujo (1964),que desde sus teorías plantea cómo los seres humanos necesitan de una educación permanente para ser libres y emancipados en cualquier realidad social que pueda ser abordada, siendo una reflexión para despertar conciencia en los seres humanos. Una comunidad que no se educa vive en la opresión de los otros, no tiene la capacidad de expresar sus opiniones ante los demás, según el autor las personas son analfabetas por falta de oportunidades para desarrollarse como personas autónomas y constructoras de su comunidad.

\section{Pregunta de investigación}

La pregunta planteada a continuación tiene presente el tema investigado sobre la educación en el adulto mayor qué piensa y cuáles serían los beneficios encontrados al continuar aprendiendo conocimientos que le son útiles para la vida. Por ello nos preguntamos:

¿Cual es la percepción del adulto mayor en relación con la educación? 


\section{OBJETIVO GENERAL}

Indagar sobre la percepción que tiene un grupo de adultos mayores de la localidad de Suba sobre la educación para plantear estrategias que promuevan el interés por el aprendizaje.

\section{OBJETIVOS ESPECÍFICOS}

Describir los discursos mostrados por un grupo de adultos mayores basados en la opinión que tienen de la educación.

Identificar los propósitos que tienen este grupo de adultos para seguir estudiando y qué les gustaría aprender.

Proponer alternativas que cumplan las expectativas que busca el adulto mayor sobre las preferencias de educación.

\section{MARCO METODÓLÓGICO}

\section{Enfoque utilizado}

Esta investigación es de corte cualitativo ya que permitió interactuar con los sujetos de estudio, proporcionando mayor profundidad de respuesta y comprensión de la realidad y está basada en experiencias humanas. Bonilla (1989), dice que "permite al investigador hacer una aproximación global de las situaciones sociales para explorarlas, describirlas y comprenderlas de manera inductiva y no deductivamente, parte de conocimientos que tienen las diferentes personas involucradas" esta idea aplica a este trabajo porque consintió la interacción con el objeto de estudio en su contexto, a través de la observación, el dialogo, palabras, discursos, expresiones verbales y no verbales como también los conocimientos de estos adultos y de su realidad, permitiendo la recolección de datos para la investigación.

\section{Tipo de investigación}

Esta investigación es de tipo exploratorio, según Dankhe (citado por, Hernández 1977). "Permite tener un grado de familiaridad con fenómenos relativamente desconocidos, obtener información completa sobre un contexto particular de la vida real". En la investigación realizada se logró hacer una exploración sobre la percepción del adulto mayor frente a la educación en esta etapa de la vida, lo cual permitió tener una aproximación a través de los resultados sobre lo que piensa cada uno de estos adultos.

\section{Participantes}

Se tomó a un grupo de 30 personas adultas mayores residentes de la localidad número 11 de suba Distrito Capital, Bogotá, ubicada al noroccidente de la ciudad, estratos 2 y 3 con diferentes niveles educativos y con edades que oscilan entre los 59 y los 79 años de edad. El 
$54 \%$ de la edad de esta población está entre los 59 y 62 años, seguida por un $27 \%$ que está entre los 65, 6 y 68,9, un 13\% está entre los 62,3 y 65,6 años, el 3\% está entre los 68,9 y 72,2 años y finalmente otro $3 \%$ está en una edad de 75 y 79 años y no tenemos ninguna persona entre los 72 y 75 años de edad.

\section{Técnicas utilizadas para recopilar la información}

a) Entrevista semiestructurada: la entrevista se le realizó a un grupo de 30 personas y consta de cinco preguntas abiertas, con cuyas respuestas se busca conocer la percepción de la educación del adulto mayor lo cual permitió acceder a los diferentes discursos sobre el tema investigado para luego analizarlos.

b) Observación directa: se utilizó como método ya que se pudo aprovechar la interacción con el entrevistado, permitiendo evaluar las diferentes actitudes verbales y no verbales frente al tema y la reacción en su discurso, como también el contexto de la entrevista así se complementó lo obtenido en el discurso a través de las entrevistas, ayudando en el análisis de la información.

c) Grupo focal: visto como una técnica grupal, se tomó como apoyo para encontrar diferentes puntos de vista del grupo de adultos mayores en relación al tema de la educación.

\section{RESULTADOS}

Como parte de los resultados obtenidos a través de la entrevista se encuentran los datos demográficos, están presentados en porcentajes para mejor visibilización.

a) El grupo de adultos mayores se encuentra entre los rangos de edades representadas a continuación:

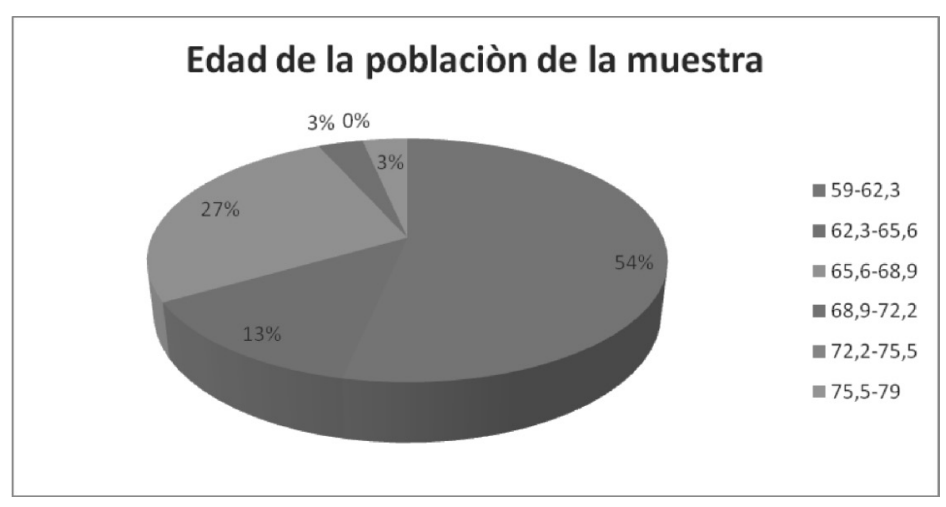

Gráfica 1. Edad de la población 
b) Nota. El grupo de adultos mayores se divide en dos grupos de 15 personas. La población está representada por edad.

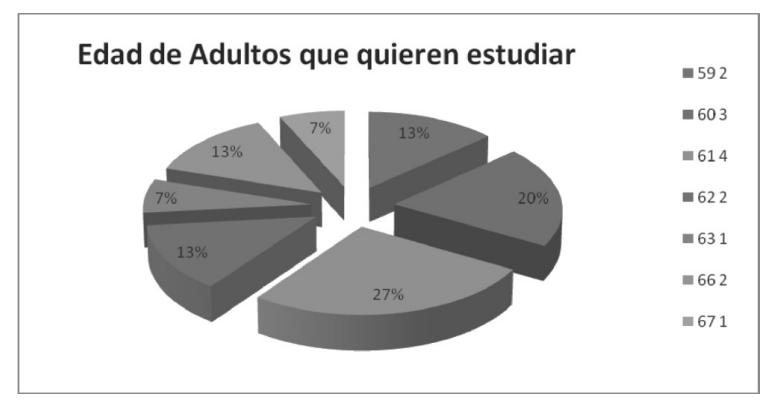

Gráfico 2. Edad de adultos que quieren estudiar

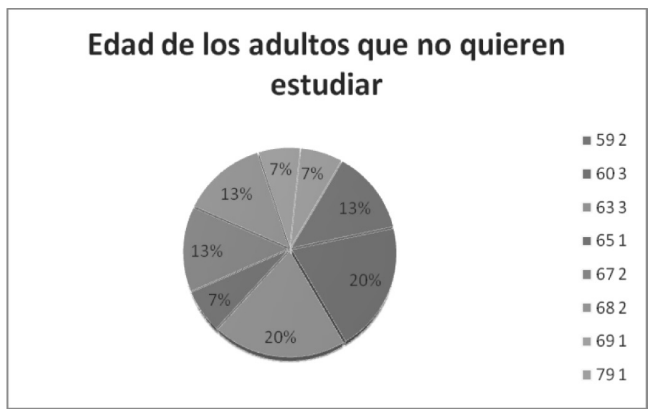

Gráfico3. Edad de adultos que no quieren estudiar

c) Nota. La siguiente clasificación del total del grupo de 30 adultos mayores está dada con datos cualitativos, de acuerdo con preferencias de aprendizaje.

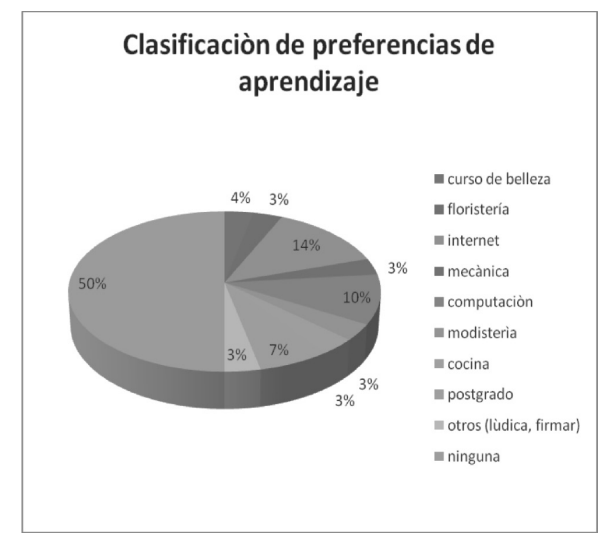

Gráfico 4. Clasificación de preferencias de aprendizaje 
d) Nota. La siguiente clasificaciòn representa el nivel educativo que tiene el grupo de adultos mayores entrevistados.

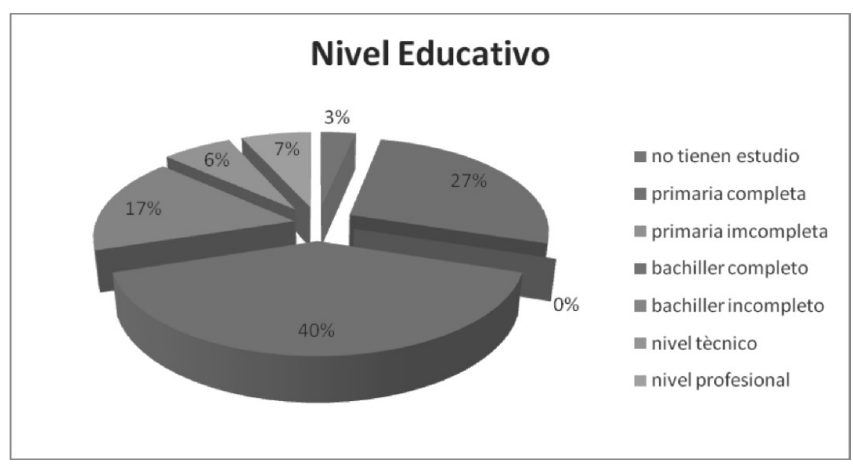

Gráfico 5. Nivel educativo

e) Nota. Esta gráfica representa el estado civil del grupo de adultos mayores de la localidad de suba.

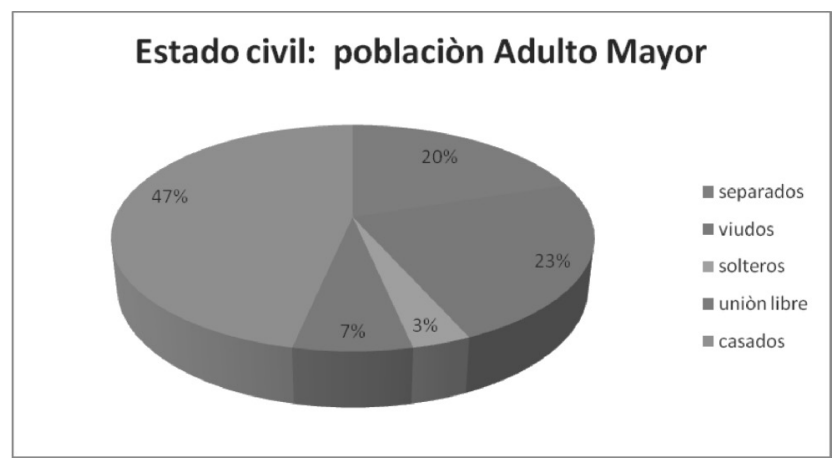

Gráfico 6. Estado civil: población adulto mayor

Tabla 1. Categorías de análisis frente al discurso

\begin{tabular}{|l|l|}
\hline CATEGORÍA GENERAL (DEDUCTIVA) & \multicolumn{1}{|c|}{ SUBCATEGORÍAS } \\
\hline EDUCACIÓN & Educación formal \\
\hline PROYECTO DE VIDA & Calidad de vida \\
RED DE APOYO FAMILIAR & Definición de apoyo familiar \\
\hline
\end{tabular}




\begin{tabular}{|l|l|}
\hline \multicolumn{1}{|c|}{ APRENDIZAJE } & \multicolumn{1}{c|}{ Creencia sobre el aprendizaje } \\
\hline MEMORIA & Limitaciones percibidas \\
\hline SENSACIÓN Y PERCEPCIÓN & Percepción de Habilidades sensoriales \\
\hline ATENCIÓN & Factores internos \\
\hline DIFERENCIAS DE APRENDIZAJE & Ideas cognitivas \\
\hline
\end{tabular}

ANÁLISIS Y DISCUSIÓN DE RESULTADOS

Freire (1964), en su obra "La educación como práctica para la libertad" mostró que una persona que tiene la oportunidad de educarse es una persona que se hace libre. En este caso, en los adultos mayores entrevistados, se evidencia que les hizo falta más oportunidades para educarse con el fin de complementar sus proyectos de vida. Desde las teorías del autor, es una reflexión para que el adulto mayor, piense en una educación sin límite de edad, raza, condición social, religión, etc.

Ahora bien, en este orden de ideas la construcción de aprendizajes y experiencias de vida en cada adulto mayor les permite tener conocimientos empíricos para toda la vida. A través de las vivencias han construido su cognición, algunos hablan de la educación como algo inalcanzable otros como una necesidad para incluirse en un medio laboral, tan sólo unos pocos no lo ven necesario, creen que han trabajado toda una vida para vivir, alimentarse y suplir sus necesidades básicas en familia.

Ahora lo que quiere el 50\% del grupo de adultos mayores con respecto a la educación son oportunidades para educarse pero no existen políticas públicas ofrecidas por la Alcaldía de Bogotá y en especial la Localidad de Suba, donde ellos puedan exigir. Según el artículo 7, programas: literal 4 y 5, sólo es dirigida hacia los niños, niñas y jóvenes; específicamente no cuentan con ningún plan de desarrollo basado en la educación para adulto mayor; sus ofertas para proteger al adulto mayor van dirigidas a la implementación de programas cortos, como son: recreación, manualidades, socialización y bonos de alimentos para los más desprotegidos; esto sustentado en el proyecto llamado "Toda la vida integralmente protegidos", dedicado a los años dorados que tiene la localidad y en general el distrito, es el planteado en el documento "Plan de Desarrollo Local Suba 2009-2012, por el cual se adopta el Plan de Desarrollo Económico, Social y de Obras Públicas para la Localidad 11 de Suba, realizado el septiembre 5 del 2008.

Pues bien, este grupo de adultos mayores demuestra en las entrevistas que es necesario que se les dé oportunidad de espacios educativos, de educación para el trabajo y educación informal, para que el adulto mayor pueda realizar proyectos que generen satisfacción personal y aumenten la calidad de vida, no solamente social sino psicológica desde una proyección educativa de formación.

A partir de investigaciones y teorías que justifican la necesidad de un aprendizaje y una educación permanente para el bienestar psicológico y social, ver el relato del adulto mayor y la 
justificación que dan en relación con sus capacidades para iniciar una etapa en áreas educativas. Aquí se plantea el tema de los procesos de aprendizaje del adulto mayor. Acorde con su discurso no quieren hacer una educación formal, no se quieren vincular con una educación que genere esfuerzos cognitivos, puesto que a ellos se les dificulta mucho leer y memorizar por la edad, además de que están cansados para ese tipo de actividades educativas y de aprendizajes que les demande lectura.

En relación con este discurso se confirman los postulados de Abarca y otros (2008) quienes, en la investigación planteada "Relación entre Educación, envejecimiento y Deterioro Cognitivo", encontraron que no sólo hay un incremento de enfermedades neurodegenerativas asociadas con la edad sino que el deterioro cognitivo aumenta en aquellas personas que no han logrado niveles educativos significativos en sus vidas o que están en el analfabetismo. Por otro lado, está Agramonte y otros (2002), quienes en la investigación "Percepción del aprendizaje desde el adulto mayor" realizada en Cuba, encontraron que en los adultos mayores no existen pérdidas ni deterioros significativos en las capacidades psíquicas, pero que sí existían necesidades de aprendizaje en el adulto mayor.

Reflexionar desde este punto de vista es tener una mirada hacia un futuro, lo que le espera a nuestra sociedad, entonces ¿qué nos espera como jóvenes enfrentar? Si el adulto mayor no quiere ser extensivo en sus procesos de aprendizaje. Se espera que a través de este tipo de investigaciones la sociedad en que está inmersa esta población adulta se dé cuenta de que hay que tenerlos en cuenta, generando desde lo social y cultural programas que les sirvan para aprehender conocimientos, para que se relacionen con otros, para mantener sus procesos cognitivos activos con el fin de que culminen una vejez digna y llena de bienestar.

\section{CONCLUSIONES}

En este grupo de adultos mayores se logró indagar la manera como ellos perciben la educación en esta etapa de la vida, a través de sus discursos expresaron sus pensamientos relacionados con el tema de la educación. Asimismo esta información se profundizó a través de dos matrices de análisis categorial; en la primera se tuvieron en cuenta dos categorías de educación: proyecto de vida y red de apoyo familiar, la segunda contiene categorías sobre los procesos de aprendizaje y las diferencias en el aprendizaje. Estos datos se organizaron para lograr interpretarlos y comprender qué es lo que les gustaría aprender.

Al describir los discursos relatados se encontró que los adultos mayores no tienen en sus proyectos de vida la educación como base fundamental para su desarrollo, además, no cuentan con los recursos ni el tiempo para dedicarse a estudiar en estos momentos, para ellos no es una necesidad básica en la vida el tema de la educación, piensan en un tipo de educación informal aquella que no requiere de mayor esfuerzo intelectual ni económico, aunque el 50\% del grupo no quiere ningún tipo de formación educativa, pues no lo ven necesario.

El pensamiento y la percepción que tienen este grupo de adultos mayores frente a la educación es así: el 50\% del grupo no desea seguir educándose, piensa que la educación en personas 
adultas mayores como ellos no serviría para nada, eso sería como para pasar el tiempo, hacen énfasis en disfrutar de la familia y nietos colaborándoles, no ven la educación como prioridad aunque les hubiera gustado haber estudiado en su juventud, piensan en la educación como un medio para el trabajo, que les sirva para tener solvencia económica para la familia, mas no para que esto les genere un bienestar tanto en su salud mental como física, conllevándolos a culminar con una calidad de vida saludable, en cuanto a su procesos cognitivos.

Por otra parte, el otro 50\% del grupo de adultos mayores piensa en la educación una oportunidad como algo positivo, además de que en algunos de ellos fue un sueño que no pudieron hacer realidad, creen que la educación es necesaria, más aún cuando se está joven para poder hacer mejores cosas ya que sin educación se pierden muchas oportunidades en la vida, piensan que si les ofrecieran una educación gratuita y en horarios flexibles sería la oportunidad de hacer cosas que no pudieron cuando jóvenes.

Se identificaron propósitos educativos o preferencias de aprendizaje en este grupo de adultos mayores sobre lo que les gustaría a prender de la siguiente manera: el $4 \%$ cursos de belleza, el 3\% cursos de floristería, el 14\%, aprender el manejo de internet, el 3\% cursos de mecánica, el 10\% cursos de computación, el 3\% modistería, el 3\% cursos de cocina, el 3\% refiere al aprendizaje pero en cursos para la recreación como baile y otras actividades lúdicas que les sirva para interactuar con otros y un 3\% no tienen ninguna preferencia. Es relevante tener en cuenta las edades de este $50 \%$ de adultos que quieren estudiar, el $27 \%$ es de 61 años, el $20 \%$ con edad de 60 años, un $13 \%$ está entre los 69 años, otro $13 \%$ de 62 años, un 13\% de 66 años, teniendo un 7\% de 63 años y otro 13\% de 67 años. Estas personas piensan que educarse ahora sería en algo que les ayude a capacitarse en conocimientos en cualquier arte que escojan y que les permita generar ingresos. En estas etapas de la vida la educación para ellos es algo productivo, ven la educación como inclusión social, sintiéndose valorados por sus saberes y experiencia obtenida a lo largo de la vida.

Las alternativas propuestas sobre educación en estos adultos mayores serían desde la educación no formal que es lo que a ellos les ha interesado, de acuerdo con sus preferencias como capacitación en diferentes cursos a corto plazo, con énfasis en el manejo lúdico de aprendizaje, con el fin de mantenerlos visibles a nivel social, como también para darles la oportunidad de ser personas activas desde lo psicológico en todas sus funciones cognitivas, generando en ellos bienestar al finalizar las etapas del desarrollo. Se infiere que estos adultos no tienen una cultura educativa a nivel cultural y social desde los imaginarios sociales.

\section{RECOMENDACIONES}

Después de haber realizado este trabajo investigativo con adultos mayores, y de analizar la información recogida a través de la metodología utilizada, la cual permitió tener un acercamiento a esta población, es importante que se sigan trabajando estos temas desde lo social comunitario en este caso la educación en el adulto mayor y muchos otros que aún competen a esta población relegada por la sociedad en esta etapa de sus vidas. 
La UNAD como institución educativa y desde su enfoque comunitario debería interesase más por temas como estos, dirigidos al adulto mayor y generar programas, donde esta población adulta sea tenida en cuenta en cuanto a los aprendizajes, que busquen bienestar en la salud mental. Sería interesante que ofertaran cursos que llamen la atención de los adultos por aprender, es decir desde la educación no formal (artes, manualidades, de recreación como aprendizaje de bailes, programas culturales que les sirva para relacionarse con sus pares) para que esta población descubra que aún siguen siendo activos a nivel sociocultural, sientan que la sociedad todavía los tiene en cuenta y se preocupa por ellos.

El trabajo sobre el adulto mayor es una realidad de la Universidad; durante dos años se ha construido un marco teórico, se ha trabajado con red social y mesas de trabajo de localidades de Bogotá como Soacha y Bosa, teniendo presente que se ha recogido información importante de conocimientos del adulto mayor y es por esto que la investigación debe tomar un horizonte dirigido a la práctica que desde la universidad, a nivel de política pública, se gestione este trabajo en beneficio de esta población adulta mayor.

\section{REFERENCIAS BIBLIOGRÁFICAS}

CARRANZA, Antonio y ROGER, Díaz, (2000), Lucha por la educación de los adultos.

DA COSTA, Salvador, (2006), Tecnología Educacional y calidad de la Educación en América Latina opción de transformación.

GERENA, Ruby Maritza, GARCÍA, Del Carmen, (2009), Los Sofistas de la edad de plata. Ed. UNAD, primera edición.

BONILLA, E. CASTRO P. RODRÍGUEZ, (1997), Mas allá del dilema de los métodos, la investigación en ciencias sociales, editorial norma.

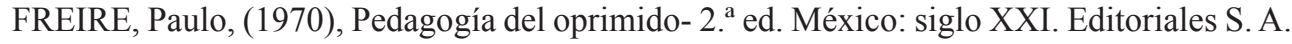
de C.V, 2005. 248p: 2k 14 Em. (Educación), primera edición.

FREIRE, Paulo (1969), La educación como práctica para la libertad. Primera edición tierra nueva Montevideo.

FERNAN, Robert (1998), Psicología con aplicaciones a los países de habla hispana, 6 ediciones, México: McGraw-Hill, psicología, percepción, inteligencia, conducta.

HOMBRADOS, María Isabel, GARCÍA Miguel, (2006) Intervención Social y Comunitaria, Ediciones Aljibe Málaga.

L. PALOMINO, Martha, H. RÍOS, Gloria y SAMUDIO, Díaz Jaime. "Proyectos de vida y rehabilitación de delincuentes” Revista Latinoamericana de Psicología. Vol. 23. n.o 1. Bogotá 1991. 
LEV, Vigostky, (2008). Pensamiento y Lenguaje. México. Ediciones Quinto sol.

MORÍN Edgar, (2002). Los siete saberes necesarios para la educación del futuro. Buenos Aires: Nueva Visión.

MORÍN, Edgar (2002), Paidos Ibérica. Los siete saberes necesarios a la educación del futuro.

REVISTA NEUROPSICOLOGÍA. Neuropsiquiatría y Neurociencias, Octubre 2008, Vol.8, n.o 2, pp. 1-9.

SCHIFFMAN, Harvey Richard (1983). La Percepción Sensorial. México D. F.: Limusa, 1983. 